\title{
NONLINEAR MAXWELL EQUATIONS
}

\author{
G.A. Kotel'nikov \\ 123182, RRC "Kurchatov Institute", Moscow, Russia \\ e-mail: kga@kga.kiae.su
}

\begin{abstract}
A new relativistic invariant version of nonlinear Maxwell equations is offerred. Some properties of these equations are considered.
\end{abstract}

The nonlinear equations of theoretical and mathematical physics attract the significant attention because of the specific properties such, as the absence of the superposition principle, the nonlinear fields interactions, existence of the soliton solutions. The nonlinear equations in electrodynamics were offered for the first time by Born [1], Born and Infeld [2], and also Schrödinger [3] outcoming from the variational principle. Later Fuschich and Tsifra 四, Fuschich [5], Fuschich, Tsifra and Bouko [6] have applied to this goal the theoretic-algebraic approach. The purpose of the present work is the formulation of Lorentz and Poincaré-invariant equations with the help of the variable replacement method.

Let us introduce the one dimensional Lorentz transformations [7]

$$
x_{1}^{\prime}=\frac{x_{1}-\beta c t}{\sqrt{1-\beta^{2}}} ; x_{2}^{\prime}=x_{2} ; x_{3}^{\prime}=x_{3} ; t^{\prime}=\frac{t-\beta x_{1} / c}{\sqrt{1-\beta^{2}}}
$$

Here $x_{1,2,3}=x, y, z ; c$ is the speed of light; $t$ is the time; $\beta=V / c ; V$ is the velocity of movement of inertial frame $\mathrm{K}^{\prime}$ relative to $\mathrm{K}$.

One can see by calculation that the nonlinear equations

$$
\begin{aligned}
& \Phi_{1}\left(I_{1}, I_{2}\right) \nabla . \mathbf{E}=4 \pi \rho ; \Phi_{1}\left(I_{1}, I_{2}\right)\left(\nabla \times \mathbf{H}-\frac{1}{c} \partial_{t} \mathbf{E}\right)=+4 \pi \rho \mathbf{v} ; \\
& \Phi_{2}\left(I_{1}, I_{2}\right) \nabla . \mathbf{H}=4 \pi \mu ; \Phi_{2}\left(I_{1}, I_{2}\right)\left(\nabla \times \mathbf{E}+\frac{1}{c} \partial_{t} \mathbf{H}\right)=-4 \pi \mu \mathbf{w}
\end{aligned}
$$

are invariant relative to transformations (1), if the values entering into them will be transformed in the known way [7]:

$$
\begin{gathered}
E_{1}^{\prime}=E_{1} ; E_{2}^{\prime}=\frac{E_{2}-\beta H_{3}}{\sqrt{1-\beta^{2}}} ; E_{3}^{\prime}=\frac{E_{3}+\beta H_{2}}{\sqrt{1-\beta^{2}}} ; \\
H_{1}^{\prime}=H_{1} ; H_{2}^{\prime}=\frac{H_{2}+\beta E_{3}}{\sqrt{1-\beta^{2}}} ; H_{3}^{\prime}=\frac{H_{3}-\beta E_{2}}{\sqrt{1-\beta^{2}}} ; \\
\rho^{\prime}=\rho \frac{1-v_{1} V / c^{2}}{\sqrt{1-\beta^{2}}} ; \\
\mu^{\prime}=\mu \frac{1-w_{1} V / c^{2}}{\sqrt{1-\beta^{2}}} ;
\end{gathered}
$$




$$
\begin{gathered}
v_{1}^{\prime}=\frac{v_{1}-V}{1-v_{1} V / c^{2}} ; v_{2,3}^{\prime}=v_{2,3} \frac{\sqrt{1-\beta^{2}}}{1-v_{1} V / c^{2}} ; \\
w_{1}^{\prime}=\frac{w_{1}-V}{1-w_{1} V / c^{2}} ; w_{2,3}^{\prime}=w_{2,3} \frac{\sqrt{1-\beta^{2}}}{1-w_{1} V / c^{2}}
\end{gathered}
$$

Here $E, H$ are the electric and magnetic fields; $\rho, \mu$ are the densities of electromagnetic charges; $v, w$ are the charge velocities; $\Phi_{1}, \Phi_{2}$ are arbitrary functions of Lorentz and Poincaré invariants of fields $I_{1}=2\left(\mathbf{E}^{2}-\mathbf{H}^{2}\right), I_{2}=(\mathbf{E} . \mathbf{H})^{2}$ [3]. In proofing the invariance of the equations it is necessary to take into account: the invariance of the speed of light; the transformation properties of the electromagnetic fields and charge densities; the law of transformation of velocities; the invariance of the functions $\Phi_{1}$ and $\Phi_{2}$.

Because of arbitrariness of the functions $\Phi_{1}$ and $\Phi_{2}$ system (2) contains the infinite set of particular realizations of nonlinear Maxwell equations, among which it is possible to indicate the following base versions:

- the linear free Maxwell equations [3] with $\rho=\mu=0$;

- the linear one-charge Maxwell equations [3] with $\Phi_{1}=\Phi_{2}=1, \mu=0$;

- the linear two-charge Maxwell equations [8] with $\Phi_{1}=\Phi_{2}=1$.

Let us note some general properties of these nonlinear equations induced.

The equations (2) become not only relativistic, but also conformal invariant, if the functions are $\Phi_{1}\left(I_{1}^{2} / I_{2}\right), \Phi_{2}\left(I_{1}^{2} / I_{2}\right)$. The statement results from the proof of conformal symmetry of linear Maxwell equations and identical conformal dimension of the values $I_{1}^{2}$ and $I_{2}$.

Equations (2) become linear in absence of currents and charges and so contain the classical electrodynamics of the free fields.

Generally, the nonlinearity is conditioned by currents and charges.

The equations keep the possibility of electromagnetic field definition through the two-potentials $A^{a}=(\phi, \mathbf{A}), B^{a}=(\Phi, \mathbf{B}), a=0,1,2,3$ [9]

$$
\mathbf{E}=-\nabla \phi-\partial_{t} \mathbf{A} / c-\nabla \times \mathbf{B} ; \mathbf{H}=-\nabla \Phi-\partial_{t} \mathbf{B} / c+\nabla \times \mathbf{A}
$$

The two-potentials satisfy the nonlinear D'Alembert equations

$$
\Phi_{1}\left(I_{1}, I_{2}\right) \square A^{a}=4 \pi J^{a} ; \Phi_{2}\left(I_{1}, I_{2}\right) \square B^{a}=4 \pi K^{a}
$$

under condition of the relativistic invariant calibrations $\partial_{a} A^{a}=0, \partial_{a} B^{a}=0$, where $\partial_{a}=\partial / \partial x^{a}, x^{0}=c t, x^{1,2,3}=x, y, z ; g_{a b}=\operatorname{diag}(+,-,-,-) ; J^{0}=\rho, \mathbf{J}=\rho \mathbf{v} / c ;$ $K^{0}=\mu, \mathbf{K}=\mu \mathbf{w} / c$. Similarly to the initial equations (2), the free equations (10) automatically become linear. 
In the important particular case of electrostatic charges in the one-charge electrodynamics with $\mathbf{A}=0$ the scalar potential $\phi$ satisfies the nonlinear Laplace-Poisson equation

$$
\Phi_{1}\left((\nabla \phi)^{2}\right) \triangle \phi=-4 \pi \rho(x)
$$

Putting here the Fourier-decomposition of the potential $\phi=(2 \pi)^{-3} \int \phi_{k} \exp (i \mathbf{k} \cdot \mathbf{x}) d^{3} k$ in case of electrical charge with the density $\rho(x)$ for the component $\phi_{k}$ we have

$$
\phi_{k}=\left(4 \pi / k^{2}\right) \int \rho(x) F_{1}\left((\nabla \phi)^{2}\right) \exp (-\mathbf{k} \cdot \mathbf{x}) d^{3} x=\left(4 \pi / k^{2}\right)\left(\rho F_{1}\right)_{k}
$$

Here $\left(\rho F_{1}\right)_{k}$ means the form-factor characterizing the electricity distribution in the effective charge $Q=\int \rho F_{1} d^{3} x, F_{1}=1 / \Phi_{1}$. The form-factor can differ from unit. This will mean the availability of corrections to the Coulomb field of a charge.

Let us write the equations (2) in some other form. We divide their right parts into the functions $\Phi_{1}$ and $\Phi_{2}$, designate $1 / \Phi_{1}=F_{1}, 1 / \Phi_{2}=F_{2}$ and instead of $\rho(x)$, $\mu(x), \mathbf{J}=\rho(x) \mathbf{v} / c, \mathbf{K}=\mu(x) \mathbf{w} / c$ we take the new values

$$
\rho \rightarrow \rho(x) F_{1} ; \mu \rightarrow \mu(x) F_{2} ; \mathbf{J} \rightarrow \rho(x) F_{1} \mathbf{v} / c ; \mathbf{K} \rightarrow \mu(x) F_{2} \mathbf{w} / c
$$

We will refer the densities of charges and currents $\rho, \mu, \mathbf{J}$ and $\mathbf{K}$ to as the initial ones, and the values corresponding them do to as the effective ones. Then it is possible to say that the nonlinear microscopic equations of electrodynamics are the equations which contain the effective values of charge densities and current densities instead of the initial ones 10

$$
\begin{gathered}
\nabla . \mathbf{E}=4 \pi F_{1}\left(I_{1}, I_{2}\right) \rho ; \nabla \times \mathbf{H}-\frac{1}{c} \partial_{t} \mathbf{E}=4 \pi F_{1}\left(I_{1}, I_{2}\right) \rho \frac{\mathbf{v}}{c} ; \\
\nabla . \mathbf{H}=4 \pi F_{2}\left(I_{1}, I_{2}\right) \mu ; \nabla \times \mathbf{E}+\frac{1}{c} \partial_{t} \mathbf{H}=-4 \pi F_{2}\left(I_{1}, I_{2}\right) \mu \frac{\mathbf{w}}{c} \\
\square A^{a}=4 \pi F_{1} \mathbf{J}^{a} ; \square B^{a}=4 \pi F_{2} \mathbf{K}^{a}
\end{gathered}
$$

These equations realize the principle of self-action: the initial charges generate electromagnetic fields which in its turn influence on the initial charges, their densities and sizes up to reaching the equilibrium state with the generating fields. So, in the nonlinear versions of the Maxwell and D'Alembert equations (14), (15) the electromagnetic charges

$$
Q=\int \rho(x) F_{1}\left(I_{1}, I_{2}\right) d^{3} x ; P=\int \mu(x) F_{2}\left(I_{1}, I_{2}\right) d^{3} x
$$

receive at least partly the field nature. This property of a charge is absent in the linear electrodynamics. The effective charges $Q$ and $P$ keep the property of Lorentz-invariance owing to the invariance of functions $F_{1}$ and $F_{2}$, and are integrals of movement due to the existance of the continuity equations 


$$
\partial_{t}\left(\rho F_{1}\right)+c \nabla \cdot\left(F_{1} \mathbf{J}\right)=0 ; \partial_{t}\left(\mu F_{2}\right)+c \nabla \cdot\left(F_{2} \mathbf{K}\right)=0
$$

It followes from the equations (17) that the initial charges are not conserved. For example, we have for the electric charge $q=\int \rho d^{3} x$

$$
\partial_{t} q=-\oint \rho \mathbf{v} d s-\int\left(\partial_{t} F_{1}+\mathbf{v} \cdot \nabla F_{1}\right)\left(\rho / F_{1}\right) d^{3} x
$$

Here $d s$ is the element of the area surrounding the volume element $d^{3} x$ as usually. The change of the charge $q$ is conditioned not only by the density of current $\mathbf{j}=\rho \mathbf{v}$, but by the change of the field invariant $F_{1}\left(I_{1}, I_{2}\right)$ in time and space.

As far as the nonlinear Maxwell equations satisfy the requirement of relativistic invariance, they have the potential interest to physics. In addition to the known general theoretical questions of electrical charge stability and nature of its mass [3], it is possible to point out also the field nature of a charge and the necessity of experimental verification of the Coulomb law at short distances. The existance of equations (14) prompt us also to induce the relativistic invariant action integral in case of the one charge electrodynamics in more general form

$$
S=-m c \int \Psi_{1}\left(I_{1}, I_{2}\right) d s-\frac{1}{c} \int \Psi_{2}\left(I_{1}, I_{2}\right) A_{a} J^{a} d^{4} x-\frac{1}{16 \pi c} \int \Psi_{3}\left(I_{1}, I_{2}\right) I_{1} d^{4} x
$$

Here as usually, $m$ is the rest mass of a particle, $d s$ is the element of the interval, $A_{a}=(\phi,-\mathbf{A}), d^{4} x=c d t d x d y d z$ [7], $\Psi_{1}, \Psi_{2}, \Psi_{3}$ are the functions of relativistic invariants $I_{1}$ and $I_{2}$.

According to (19) we can indicate the six versions of Maxwell electrodynamics with the invariant speed of light:

- the classical linear electrodynamics with $\Psi_{1}=\Psi_{2}=\Psi_{3}=1$ [3], [7];

- the linear electrodynamics with $\Psi_{1} \neq 1, \Psi_{2}=\Psi_{3}=1$;

- the nonlinear electrodynamics of the first type with $\Psi_{3} \neq 1, \Psi_{1}=\Psi_{2}=1$;

- the nonlinear electrodynamics of the second type with $\Psi_{2} \neq 1, \Psi_{1}=\Psi_{3}=1$;

- the nonlinear electrodynamics of the third type with $\Psi_{2} \neq 1, \Psi_{3} \neq 1, \Psi_{1}=1$;

- the nonlinear electrodynamics of the fourth type with all functions $\Psi \neq 1$.

In particular, the Born model with $\Psi_{3}=4 E_{0}^{2}\left[1-\left(1-I_{1} / 2 E_{0}^{2}\right)^{1 / 2}\right] / I_{1}$ [回, the BornInfeld model with $\Psi_{3}=4 E_{0}^{2}\left[1-\left(1-I_{1} / 2 E_{0}^{2}-I_{2} / 4 E_{0}^{4}\right)^{1 / 2}\right] / I_{1}$ [2], the Schrödinger model with $\Psi_{3}=2 E_{0}^{2} \ln \left(1+I_{1} / 2 E_{0}^{2}\right)$ [3] belong to the nonlinear version of the first type. (Here $E_{0}$ is the maximum field [3]).

This work belongs to the nonlinear version of the second type, as far as the variation of the integral (19) with the invariable value of $\Psi_{2} J^{a}$ leads to the equations 
(14). For example, within framework of this version the nonlinear Laplace-Poisson equation (11) may be written as follows:

$$
\left[1+\alpha\left(\frac{\partial \phi}{\partial r}\right)^{2}\right]\left[\left(\frac{1}{r^{2}}\right) \frac{\partial}{\partial r}\left(r^{2} \frac{\partial \phi}{\partial r}\right)\right]= \begin{cases}-\frac{2 q}{r^{2}}\left(\frac{a}{\sqrt{\pi}} e^{-a^{2} r^{2}}\right) & \text { if } \rho=\rho_{1} \\ -\frac{2 q}{r^{2}}\left(\frac{a}{\pi} \frac{\operatorname{sinar}}{a r}\right) & \text { if } \rho=\rho_{2}\end{cases}
$$

Here we put that $\Phi_{1}=\left[1+\alpha I_{1}\right]=\left[1+\alpha \mathbf{E}^{2}\right]_{H=0}=\left[1+\alpha(\partial \phi / \partial r)^{2}\right] ; \rho_{1}=$ $\left(q / 2 \pi r^{2}\right)(a / \sqrt{\pi}) \exp \left(-a^{2} r^{2}\right) ; \rho_{2}=\left(q / 2 \pi r^{2}\right)(a / \pi)(\operatorname{sinar} / a r), q$ is the electrical charge, $r=\left(x^{2}+y^{2}+z^{2}\right)^{1 / 2}, \alpha=k / a, k$ is the proportionality coefficient, $a$ is the parameter with inverse length dimension $(1 / \mathrm{cm})$. Tending $a \rightarrow \infty(\alpha \rightarrow 0)$, we have the linear Laplace-Poisson equation

$$
\frac{1}{r^{2}} \frac{\partial}{\partial r}\left(r^{2} \frac{\partial \phi}{\partial r}\right)=-\frac{2 q}{r^{2}} \delta(r),
$$

where the solution of the equation has the known form $\phi=q / r$. One can see, that the linear theory is the theory with point-charges. The nonlinear theory is the theory with the density of charges distributed over space. Densities of charges $\rho_{1}$ and $\rho_{2}$ correspond to the various physical models of charges.

The others versions were not investigated. They can be accompanied by the dependence of the effective mass on the electromagnetic field.

In addition to these six versions, it is possible to formulate the nonlinear electrodynamics with the noninvariant speed of light. The model of this type was proposed by Fushchich [0].

\section{References}

[1] M. Born. Proc. Roy. Soc. A, 1934, v. 143, N 848, p. 410-437.

[2] M. Born, L. Infeld. Proc. Roy. Soc. A, 1934, v. 144, N 850, p. 425-451.

[3] D. Ivanenko, A. Sokolov. Classical Theory of Field. oscow-Leningrad: Gostexizdat, 1951, p. 199.

[4] W.I. Fuschich, I.M. Tsifra. Teor. Mat. Fizika (Russia), 1985, v. 64, N 1, p. 41-50.

[5] W.I. Fuschich. Dok. Acad. Nauk (Ukraina), 1992, N 4, p. 24-27.

[6] W. Fuschych, I. Tsyfra, V. Bouko. Nonlinear Math. Phys. (Ukraina), 1994, v.1, N 2, p. 210-221.

[7] L.D. Landau, I.M. Lifshits. Theory of Field. oscow: Nauka, 1973, p. 89, 102.

[8] V.I. Strazhev, L.M. Tomilchik. Electrodynamics with Magnetic Charge. Minsk: Nauka i Technika, 1975, p. 18, 41. 
[9] N. Cabibo, E. Ferrari. Nuovo Cimento, 1982, v. 23, N. 6, p. 1147-1154.

[10] G.A. Kotel'nikov. Izv. VUZov (Russia), 1995, N 2, p. 116-119. 